\title{
Characterization and fine mapping of a new dwarf mutant in Brassica napus
}

Xin $\mathrm{Li}^{1,2,3 \dagger}$, Fujiang Xiang ${ }^{1,2+}$, Wei Zhang ${ }^{4 \dagger}$, Jindong Yan ${ }^{1,2}$, Xinmei $\mathrm{Li}^{1,2}$, Ming Zhong ${ }^{1,2}$, Piao Yang ${ }^{1,2}$, Caiyan Chen ${ }^{3}$, Xuanming Liu', Donghai Mao ${ }^{3^{*}}$ and Xiaoying Zhao ${ }^{1,2^{*}}$ (D)

\begin{abstract}
Background: Plant height is an important plant characteristic closely related to yield performance of many crops. Reasonable reduction of plant height of crops is beneficial for improving yield and enhancing lodging resistance.

Results: In the present study, we described the Brassica napus dwarf mutant bnd2 that was isolated using ethyl methanesulfonate (EMS) mutagenesis. Compared to wild type (WT), bnd2 exhibited reduced height and shorter hypocotyl and petiole leaves. By crossing the bnd2 mutant with the WT strain, we found that the ratio of the mutant to the $W T$ in the $F_{2}$ population was close to $1: 3$, indicating that bnd2 is a recessive mutation of a single locus. Following bulked segregant analysis (BSA) by resequencing, BND2 was found to be located in the 13.77$18.08 \mathrm{Mb}$ interval of chromosome A08, with a length of $4.31 \mathrm{Mb}$. After fine mapping with single nucleotide polymorphism (SNP) and insertion/deletion (InDel) markers, the gene was narrowed to a 140-Kb interval ranging from $15.62 \mathrm{Mb}$ to $15.76 \mathrm{Mb}$. According to reference genome annotation, there were 27 genes in the interval, of which BnaA08g20960D had an SNP type variation in the intron between the mutant and its parent, which may be the candidate gene corresponding to BND2. The hybrid line derived from a cross between the mutant bnd2 and the commercial cultivar L329 had similar plant height but higher grain yield compared to the commercial cultivar, suggesting that the allele bnd2 is beneficial for hybrid breeding of lodging resistant and high yield rapeseed.
\end{abstract}

Conclusion: In this study, we identified a novel dwarf mutant of rapeseed with a new locus, which may be useful for functional analyses of genetic mechanisms of plant architecture and grain yield in rapeseed.

Keywords: Brassica napus, Dwarf, Grain yield, BSA, Fine mapping

\footnotetext{
*Correspondence: donghai.mao@isa.ac.cn; xiaoyzhao@hnu.edu.cn

${ }^{+}$Xin Li, Fujiang Xiang and Wei Zhang contributed equally to this work.

${ }^{3}$ Key Laboratory of Agro-Ecological Processes in Subtropical Region, Institute

of Subtropical Agriculture, Chinese Academy of Sciences, Changsha 410125,

China

${ }^{1}$ College of Biology, Hunan Hybrid Rape Engineering and Technology

Research Center, Hunan University, Changsha 410082, China

Full list of author information is available at the end of the article
}

(C) The Author(s). 2021 Open Access This article is licensed under a Creative Commons Attribution 4.0 International License, which permits use, sharing, adaptation, distribution and reproduction in any medium or format, as long as you give appropriate credit to the original author(s) and the source, provide a link to the Creative Commons licence, and indicate if changes were made. The images or other third party material in this article are included in the article's Creative Commons licence, unless indicated otherwise in a credit line to the material. If material is not included in the article's Creative Commons licence and your intended use is not permitted by statutory regulation or exceeds the permitted use, you will need to obtain permission directly from the copyright holder. To view a copy of this licence, visit http://creativecommons.org/licenses/by/4.0/. The Creative Commons Public Domain Dedication waiver (http://creativecommons.org/publicdomain/zero/1.0/) applies to the data made available in this article, unless otherwise stated in a credit line to the data. 


\section{Background}

Brassica napus (rapeseed) is a major oil crop that is vital for ensuring a supply of edible oil, improving food structure, and promoting the development of aquaculture and light textile industry [1]. B. napus belongs to the Brassica oil crops of Cruciferae [2]. It is a heterotetraploid plant species formed by natural distant hybridization of two basic diploid species of $B$. rapa and B. oleracea. The genome of $B$. napus contains approximately 100,000 protein coding genes [3]. The plant exhibits increased height among the conventional rapeseed varieties, and its height is increased by more than $20 \mathrm{~cm}$ on average in the hybrid varieties due to widespread heterosis in B. napus [4]. A serious problem that framers encounter with tall plants is that they are prone to lodging. As plant height increases, it becomes a major factor restricting yield increase and mechanized harvesting of rapeseed oil [5]. More than $60 \%$ reduction in yield was reported to be caused by lodging as a result of increased plant height in B. napus [6]. Dwarfism of B. napus is crucial for increasing both lodging resistance and yield production [7]. However, dwarf phenotypes are sometimes associated with poor agronomic traits, resulting in poor yield. Therefore, it is of great value for rapeseed breeding to cultivate varieties which can be used for cross breeding with no change in plant height and that perform better in terms of yield.

To date, many dwarf mutants have been identified, but the alleles useful for breeding are rare in rapeseed. For example, the $B$. napus dwarf mutant NDF-1 was approximately $70 \mathrm{~cm}$ tall, and all agronomic characteristics except for seed weight were much less favorable than its original parents. The decrease in the number of siliques per plant as well as the number of seeds per silique led to a decline in yield [8]. Mutant bndf-1 with a height of $75 \mathrm{~cm}$ had more branches, but the plants were too short, resulting in fewer siliques and lower yield [9]. The semidwarf mutant $d s-1$ was only $69.3 \mathrm{~cm}$ high, and showed a lower yield per plant due to the decrease in the number of siliques per plant [10]. The semi-dwarf mutant $d w-1$, approximately $95 \mathrm{~cm}$ high, showed a higher number of siliques per plant, but the decreased yield per plant was due to significantly lower numbers of seeds per plant [11]. The mutant 'GRC1157 was only $\sim 90 \mathrm{~cm}$ at maturity and showed obvious reduction in main inflorescence length, silique numbers per main inflorescence, and seeds per silique [12]. The semi-dwarf mutant $d s$ - 3 with a height of $\sim 70 \mathrm{~cm}$ displayed fewer total nodes, shorter internodes and main inflorescences, and the position of the first main branch was lower than that of the wild type [13]. There were also some mutants that although shorter than wild type, their yield was unaffected. The dwarf mutant $D W$ $871 \mathrm{had}$ an average plant height of $139.1 \mathrm{~cm}$, and compared to the homologous high stem strain, had more effective first branches; however, there was no significant difference in the number of effective siliques, the number and weight of seeds per plant, nor in yield per plant [14]. The EMS-mutagenized sca mutant with a plant height of $\sim 80 \mathrm{~cm}$ was derived as a consequence of a mutation in a single semi-dominant gene, which encodes an Aux/IAA protein (BnaA3.IAA7). The mutant had more siliques per plant, with a similar thousand-seed weight, but each silique had fewer seeds resulting in a similar yield per plant compared to wild type [15]. In addition, the mutants Bndwf1 [16], ds-4 [17], and G7 [18] had heights of 80$110 \mathrm{~cm}, 23.4 \mathrm{~cm}$, and $30 \mathrm{~cm}$, respectively, but there was no more description of yield-related traits. In this study, we described the dwarf mutant bnd2 (B. napus dwarf 2) generated by EMS mutagenesis [19]. The bnd2 mutant showed a reduction in plant height, and grain yield compared to wild type. However, the hybrid line $F_{1}$ produced by crossing the mutant bnd 2 with the commercial variety L329, showed no increase in plant height, but showed an increase in grain yield compared to the variety L329, suggesting that bnd2 was a new locus for plant dwarfism and is useful for hybrid breeding of lodging resistant and high yielding in B. napus.

Bulked segregant analysis (BSA) is a rapid method used to detect molecular markers associated with target traits in mapping populations [20]. The combination of BSA and Next Generation Sequencing (BSA-seq) accelerates the cloning of genes responsible for important traits [21]. BSA-seq has been successfully used to map important agronomic traits in many crops such as rice [22, 23], potato [24], and soybean [25]. In this study, the locus bnd2 for dwarfism was primarily mapped using BSA-seq. The bnd 2 was then fine mapped into a $140-\mathrm{Kb}$ interval where the candidate gene BnaA08g20960D was identified. Our findings provide a foundation for cloning of the $B N D 2$ gene, providing a new locus for conferring lodging-resistance and hybrid breeding in $B$. napus.

\section{Results \\ Phenotypic characteristics of the dwarf mutant bnd 2}

The $B$. napus mutant, bnd2, was isolated from EMSmutagenized seeds of the cultivar " $2 \mathrm{~B}$ " (wild type, WT) [19]. At the seedling stage, bnd2 showed reduced hypocotyl length and shorter petiole leaves compared to WT (Fig. 1a-e, Additional files 1, 2 and 3: Figs. S1-3). At the flowering stage, the bnd 2 mutant exhibited an extremely dwarf and compact stature, and the flowering period of bnd2 was slightly longer than that of WT (Fig. If and g, Additional file 4: Fig. S4). At maturity, the plant height of $b n d 2$ was $100.65 \pm 8.09 \mathrm{~cm}(n=10)$, which was $59.8 \%$ of WT height $(168.2 \pm 7.61 \mathrm{~cm}, n=10)$ (Fig. $1 \mathrm{~h}$ and i, Additional file 5: Fig. S5, Additional file 6: Table S1). Furthermore, the first branch height, internode length, internode number, and main inflorescence length of bnd 2 were $41,76.7,69$, and $85.2 \%$ of that of WT, 


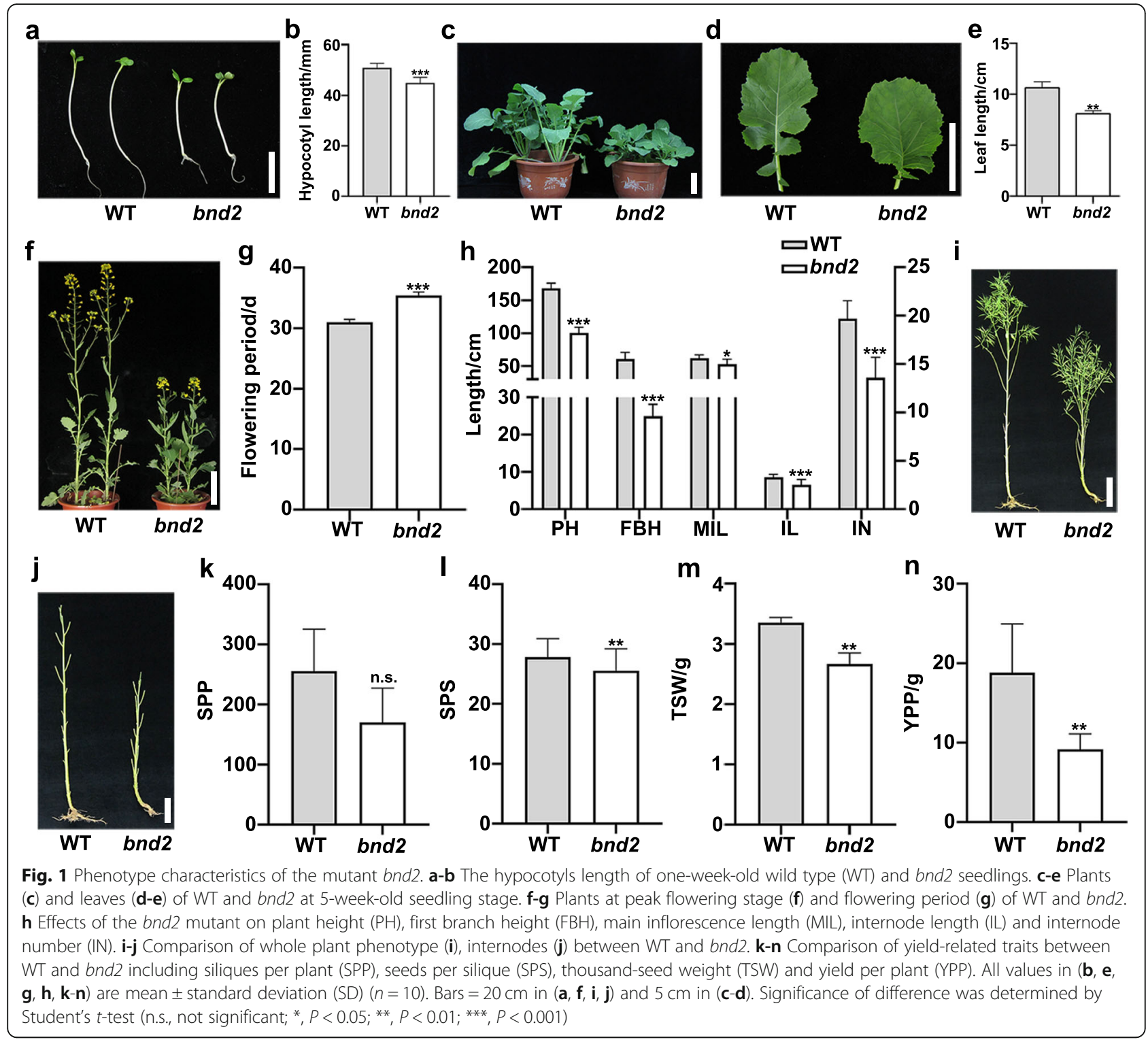

respectively. These results suggested that the dwarf traits were associated with lower position of the first branch, shorter internode length, lower internode number, and reduced main inflorescence length (Fig. 1h-j, Additional files 5 and 7: Figs. S5 and 6, Additional file 6: Table S1). Accordingly, bnd2 produced lower yield per plant (YPP) (48.4\% of WT) due to shorter silique length $(83.1 \%$ of WT), fewer seeds per silique (SPS) (92.1\% of WT), and less thousand-seed weight (TSW) (90\% of WT) compared to WT, although similar siliques per plant (SPP) were observed in both bnd 2 and WT (Fig. 1k-n, Additional file 6: Table S1, Additional file 8: Fig. S7).

Cell elongation and expansion is decreased in stems of bnd2 To investigate the underlying cellular basis for the dwarf phenotype in $b n d 2$, we performed paraffin sectioning and observed cross and longitudinal sections of the stems of bnd2 and WT at the early bolting stage. As shown in Fig. 2, the parenchymal cells of bnd 2 were arranged closely and displayed irregular shapes and different sizes compared to WT (Fig. 2a and b). The cell area and cell length were significantly reduced in both cross and longitudinal sections in the $b n d 2$ plant (Fig. 2c-f). Indeed, in cross and longitudinal sections, the cell area was decreased by 48.2 and $50.5 \%$, and cell length was decreased by 31.6 and $16.6 \%$, respectively. These results suggested that the reduction of parenchyma cell area and length in the plant stem was likely to be the main cause of the dwarfism of the mutant bnd2.

Inheritance of the dwarf phenotype in the mutant bnd 2 To analyze the inheritance of the dwarf mutant, bnd2 was crossed with its original WT parent, and with the 


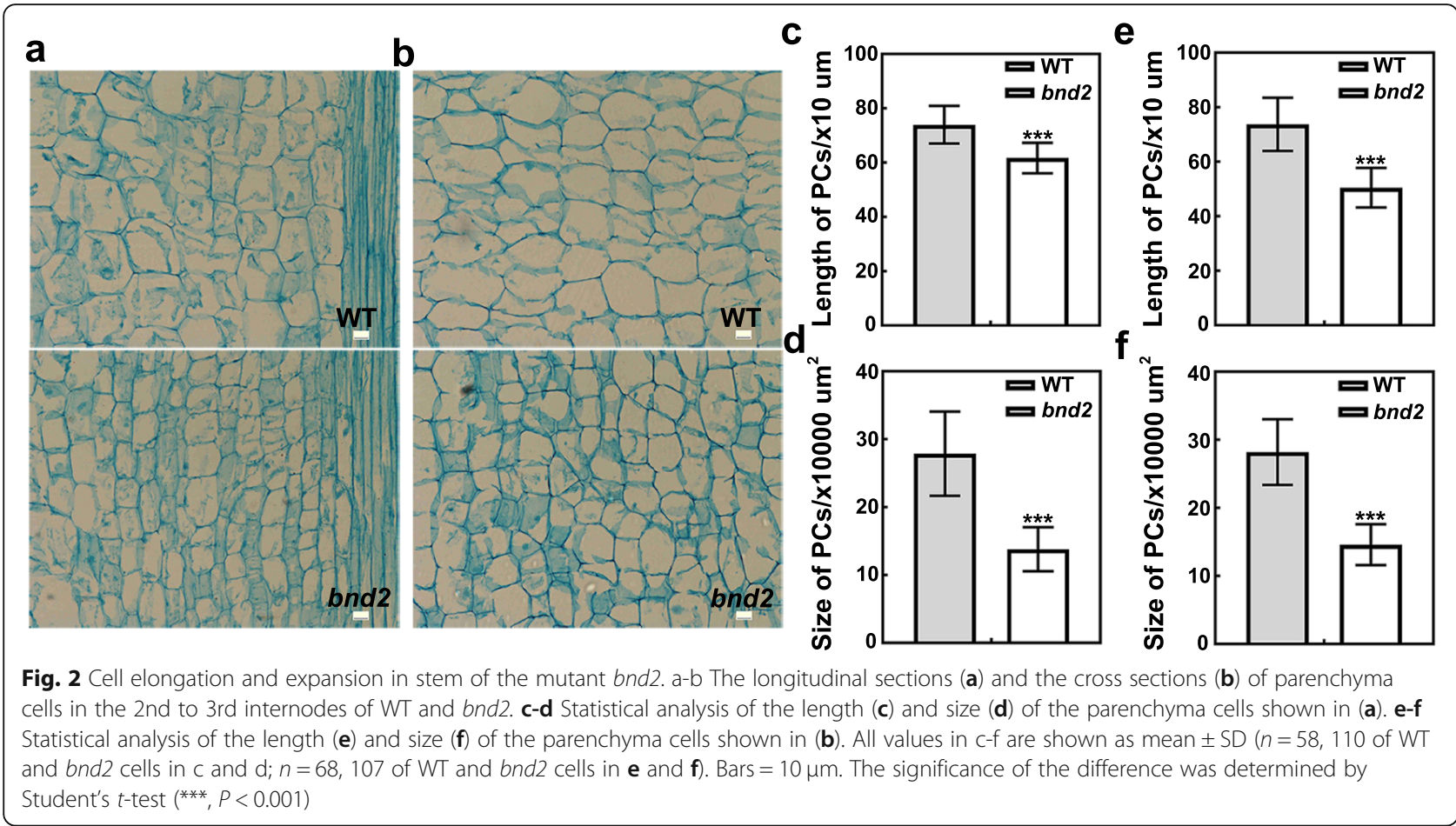

commercial cultivar L329. The resulting heterozygous $\mathrm{BC}_{1} \mathrm{~F}_{1}$ plants $(b n d 2 / \mathrm{WT})$ displayed an intermediate plant height between that of WT and the mid-parent value, suggesting that the allele $B N D 2$ is semi-dominant to the allele bnd2 (Fig. 3a-c, Additional files 9 and 10: Figs. S8 and 9). In addition, based on the plant height of the $\mathrm{BC}_{1} \mathrm{~F}_{2}$ generation of $(\mathrm{WT} \times b n d 2)$, the $236 \mathrm{BC}_{1} \mathrm{~F}_{2}$ plants were classified into two groups: the dwarf phenotype of bnd2 (dwarf plants, $n=49$ ), and the tall plant group with a height similar or close to WT (tall plants, $n=187$ ). The $\mathrm{BC}_{1} \mathrm{~F}_{2}$ generation was in line with an expected Mendelian inheritance ratio of 1:3 (dwarf plants: tall plants, $X^{2}=2.04<\chi_{0.05,1}^{2}=3.84$ ) (Fig. $3 \mathrm{~d}$ ). Another $\mathrm{F}_{2}$ population was generated from a cross between bnd2 and the commercial cultivar L329 which possessed a normal plant height of $\sim 159 \mathrm{~cm}$. There were 75 plants with a dwarf phenotype and 188 plants with plant height similar or close to that of L329 in the $F_{2}$ population, also showing a Mendelian segregation ratio of 3:1 (tall plants: dwarf plants, $\chi^{2}=1.46<\chi_{0.05,1}^{2}=3.84$ ) (Additional file 11: Fig. S10). Taken together, these results suggested that the dwarf phenotype of bnd2 was controlled by a single recessive gene.

\section{Genetic mapping of the dwarf mutant bnd 2 by BSA-seq}

To map the gene conferring the bnd 2 phenotype, the $\mathrm{F}_{2: 3}$ population derived from a cross between bnd2 and L329 was used to perform BSA resequencing. In the $\mathrm{F}_{2: 3}$ population $(n=157), 25$ extremely dwarf and 23 extremely tall homozygous lines were selected to make a short and high bulk. After sequencing the two bulks and their parents, the total data after quality control filtering was $118.75 \mathrm{~Gb}$, of which $31.21,26.11,29.09$, and $32.34 \mathrm{~Gb}$ corresponded to the L329 parent, the mutant bnd2 parent, the high bulk, and the short bulk, respectively, with a coverage depth of $24.33 \mathrm{X}, 18.55 \mathrm{X}, 21.41 \mathrm{X}$, and $22.35 \mathrm{X}$, separately (Additional file 12: Table S2). Clean reads of 105,361,953, 89,416,611, 99,097,181 and 109,214,266 were harvested for the L329 parent, the mutant bnd2 parent, the high bulk, and the short bulk, respectively (Additional file 12: Table S2). The sequencing data showed that the percentage of bases with a quality score of more than 30 (Q30) in two pools and two parents reached more than $92.99 \%$, and Q20 reached more than 97.78\% (Additional file 12: Table S2). In addition, the average GC content was $37.35 \%$, and the average genome coverage was $74.57 \%$ (Additional file 12: Table S2). Therefore, we consider that the quality of the sequencing data is consistent with expectations and can be used for further analysis. According to alignment with the 'Darmor-bzh' reference genome [26], 1,157,351 polymorphisms (containing 948,896 single nucleotide polymorphisms (SNPs) and 208,455 insertions/deletions (InDels)) were identified in the two pools. The G' value and SNP-index were calculated from the short bulk and the high bulk; the $\Delta$ (SNPindex) was drawn based on the physical positions of the reference genome (Fig. 4a and b). Only one significant $\Delta$ (SNP-index) peak was identified and located in the 4.31 


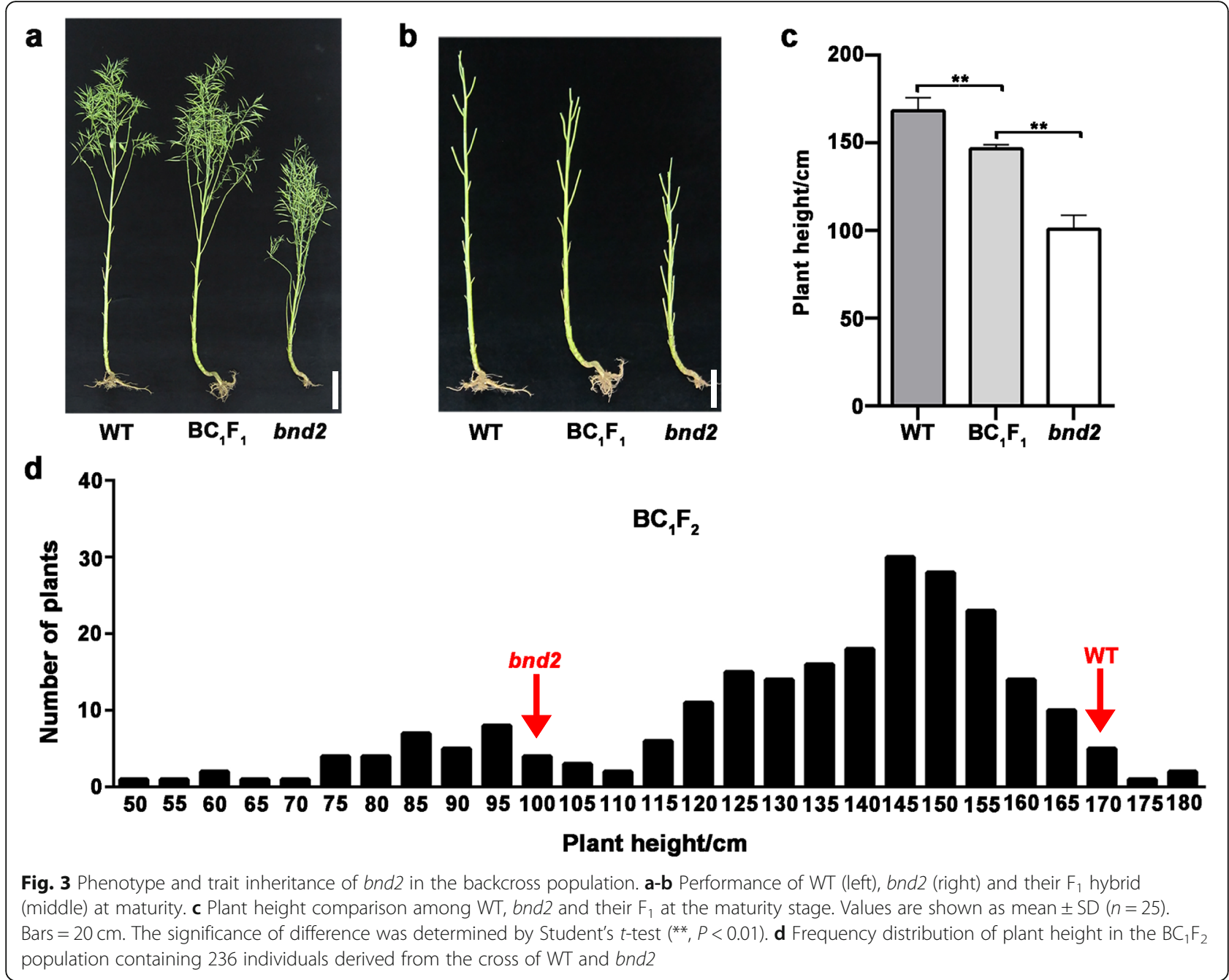

$\mathrm{Mb}$ region from $13.77 \mathrm{Mb}$ to $18.08 \mathrm{Mb}$ on chromosome A08 (Fig. 4c), suggesting that it was the candidate locus harboring the BND2 gene.

Fine mapping and candidate gene analysis

To fine map the BND2 locus, six InDel markers (ID1421, ID1470, ID1482, ID1530, ID1656, and ID1667) were developed from the $4.31-\mathrm{Mb}$ region harboring bnd2 based on the BSA-seq result. Then, in the $F_{2: 3}$ population (bnd2/L329) with 543 lines, the six markers were used to genotype 107 recessive dwarf lines, as well as two controls, including 25 WT lines and 25 heterozygous lines with segregation in plant height (Fig. 5, Additional file 13: Fig. S11). According to fine genotypes of these lines, bnd2 was further fine mapped into the 1.26$\mathrm{Mb}$ interval flanked by two InDel markers, ID1530 and ID1656 (Fig. 6a). To further narrow the candidate interval, six pairs of new polymorphic markers were developed in the region of $b n d 2$, including the SNP markers SNP1540, SNP1552, SNP1553, SNP1557, and SNP1562, and the
InDel marker ID1576 (Fig. 6b). Subsequently, BND2 was narrowed down to an interval from $15.62 \mathrm{Mb}$ to $15.76 \mathrm{Mb}$, and the physical distance was $140.0 \mathrm{~Kb}$ (Fig. 6b). After fine mapping and annotating the information of the reference genome 'Darmor- $b z h$ ', there were 27 genes in the $140 \mathrm{~Kb}$ candidate interval, 14 of which were not cloned or had unknown functions (Fig. 6c). By analyzing the annotation results of all mutations in the candidate interval, one SNP occurred in the candidate gene, BnaA08g20960D (Fig. 6d), which encodes an inositol-pentakisphosphate 2-kinase family protein, where a single nucleotide change from $\mathrm{C}$ to $\mathrm{T}$ occurred in the fifth intron region. Moreover, quantitative real-time PCR (qRT-PCR) results showed that the expression of BnaA08g20960D in bnd 2 was significantly lower than that of WT (Additional file 14: Fig. S12). Semiquantitative PCR (semi-qPCR) analysis further confirmed that BnaA08g20960D exhibited reduced mRNA expression, although no splicing changes were found in bnd2 (Additional file 15: Fig. S13). Therefore, we considered this gene as a key candidate gene. 


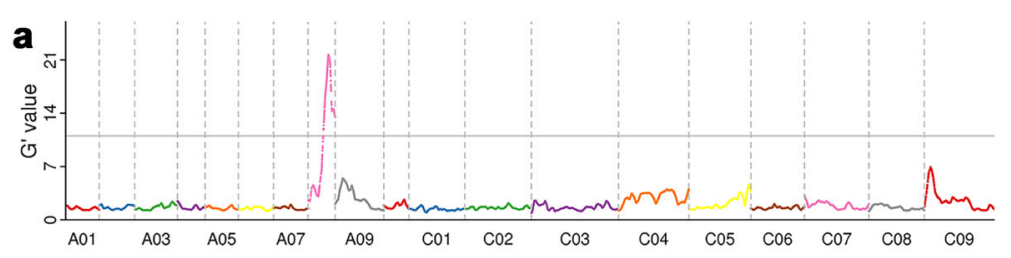

b

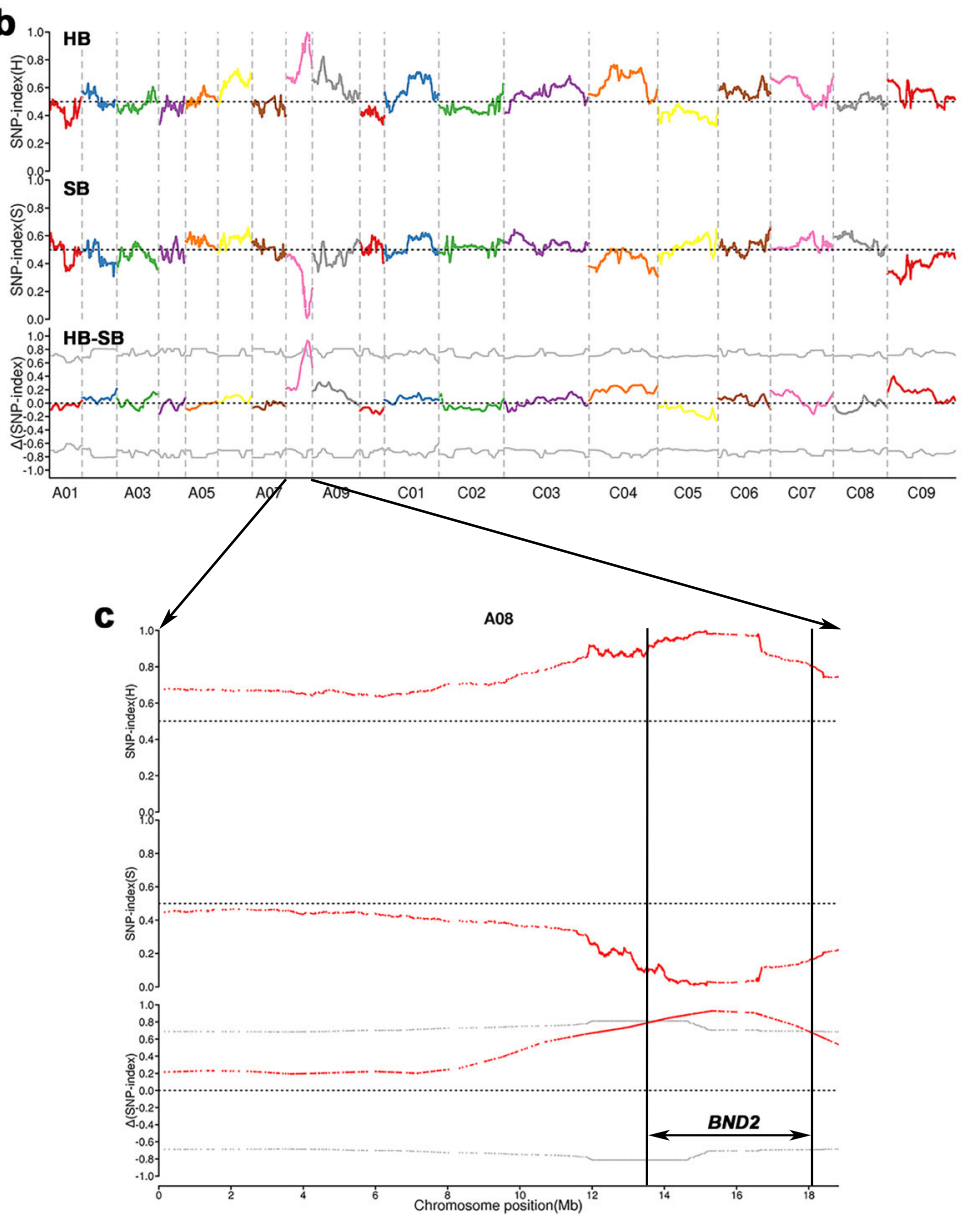

Fig. 4 Primary mapping of BND2 by bulked segregant analysis using resequencing. The physical position (unit: MB) of each chromosome in Brassica napus is represented as the $\mathrm{x}$-axis. a The $\mathrm{G}^{\prime}$-value was represented as the $\mathrm{y}$-axis. $\mathbf{b}$ The SNP index of 3-Mb interval with 10-kb sliding window each time was represented by the $y$ axis. The $\triangle$ (SNP-index) was calculated by subtracting the SNP index of the short bulk (SB) from that of the high bulk $(\mathrm{HB})$. The dotted line was the threshold of $\triangle\left(S N P\right.$-index) set as the mean of $\triangle(S N P$-index $) \pm 3^{*} S D$. $\mathbf{c}$ The BND2-containing genomic interval was identified by using the threshold line

\section{The potential application of bnd 2 in hybrid rapeseed breeding}

Due to the low yield of bnd2, it cannot be used for inbreeding of rapeseed. To test its potential application in hybrid breeding, we crossed the bnd2 mutant (bnd2/ bnd2) with the commercial cultivar L329 (BND2/BND2) to derive the hybrid line $\mathrm{F}_{1}(B N D 2 / b n d 2)$. The plant height of the $F_{1}$ hybrid was similar to L329 (Fig. 7a and b, Additional file 16: Fig. S14, Additional file 17: Table S3). However, the yield per plant (YPP) of $F_{1}$ was significantly higher than both of bnd2 and L329, showing an increase of $32.7 \%$ compared to L329 due to more seeds per silique
(SPS), and three times as much as bnd2 (Fig. 7c, Additional file 17: Table S3). These results suggested that although the presence of bnd2 in the hybrid line dose not increase plant height, it induces higher grain yield due to the semi-dominant effect of $B N D 2$ over $b n d 2$ and the heterosis between the two lines.

\section{Discussion}

Plant height is an important plant characteristic closely related to yield performance of many crops; however, very tall plants tend to have increased risk of lodging. Although many dwarf mutants in rapeseed have been 

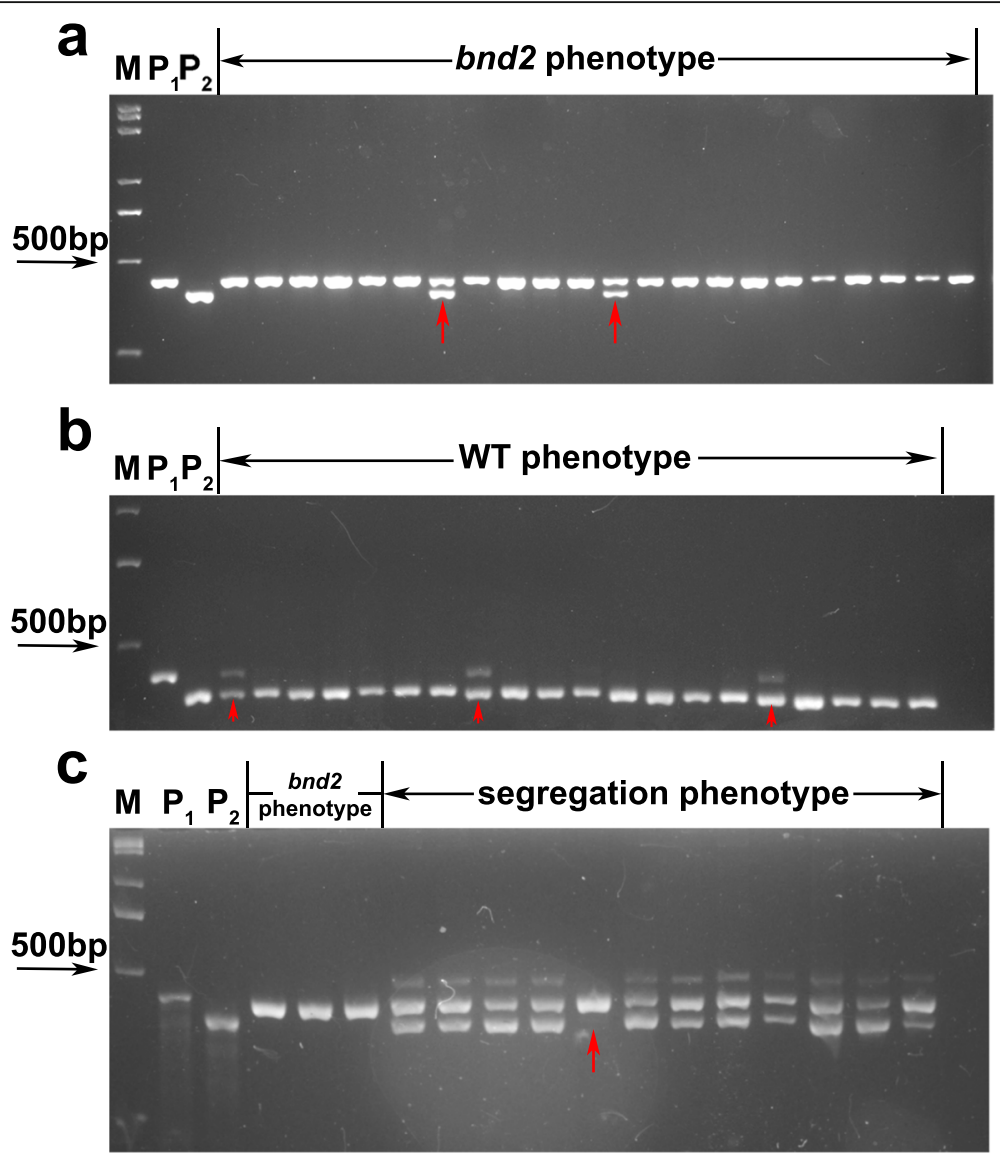

Fig. 5 The genotypes of some $F_{2: 3}$ lines derived from cross between bnd2 and L329 at the marker ID1656. a The genotypes of the lines with the bnd2 phenotype. $\mathbf{b}$ The genotypes of the lines with WT phenotype. $\mathbf{c}$ The genotype of the lines with phenotype segregation. M means DNA Marker. $P_{1}$ means the mutant parent bnd2. $P_{2}$ means the WT parent L329. The red arrow indicates the recombinants between BND2 and the marker ID1656

identified and reported, only a few varieties could be used as practical breeding resources [8-18]. Compared with rice and wheat [27], rapeseed dwarf mutants are rare. In this study, we described a new dwarf mutant bnd2 isolated from EMS-mutagenized seeds of B. napus [19]. The mutant bnd2 displayed a height of approximately $100 \mathrm{~cm}$ at maturity. The decrease in plant height was due to lower position of the first branch, shorter internodes, and reduced main inflorescence length (Fig. 1h-j, Additional files 5 and 7: Figs. S5 and 6, Additional file 6: Table S1). The reduced first branch height and main inflorescence length are conducive to lodging resistance [28]. The mutant bnd2 displayed a poor biological yield performance due to the limitation of its height (Fig. 1k-n), which limits its benefit for inbreeding of high-yield cultivars. While it was reported that a $B$. rapa dwarf mutant Brrga1-d showed significant reduction in seed yield, it had no significant influence on the seed yield of hybrid lines containing the dwarf allele in B. napus [29]. The relatively short sca mutant displayed intermediate height between corresponding parents, and significantly higher YPP after crossing it with the rapeseed cultivars 4312, ZS11, and ZY821 [15]. In this study, as shown in Fig. 3, the heterozygous $\mathrm{BC}_{1} \mathrm{~F}_{1}$ plants (bnd2/WT) derived from a backcross of bnd2 with its WT parent, displayed intermediate plant height between that of WT and the mid-parent value (Fig. 3a-c, Additional files 9 and 10: Figs. S8 and 9), suggesting that the allele BND2 is semi-dominant over the allele bnd2. While the $\mathrm{F}_{1}$ plants (bnd2/L329) derived from crossing bnd2 with the commercial cultivar L329 showed no significant difference with regards to plant height (Fig. 7a and b, Additional file 16: Fig. S14, Additional file 17: Table S3), the plants displayed a significant increase in grain yield compared to L329 (Fig. 7c, Additional file 17: Table S3), suggesting that by combining the semidominant effect of bnd 2 and the heterosis between two lines, the allele $b n d 2$ may be a potential genetic resource for lodging-resistance and high-yield breeding in hybrid rapeseed.

In the fine mapping interval of $b n d 2$, a candidate gene BnIPK1, BnaA08g20960D (Fig. 6c) was annotated to encode an inositol 1,3,4,5,6-pentapentaphosphate 2 kinase, which catalyzes the terminal step in the biosynthetic 


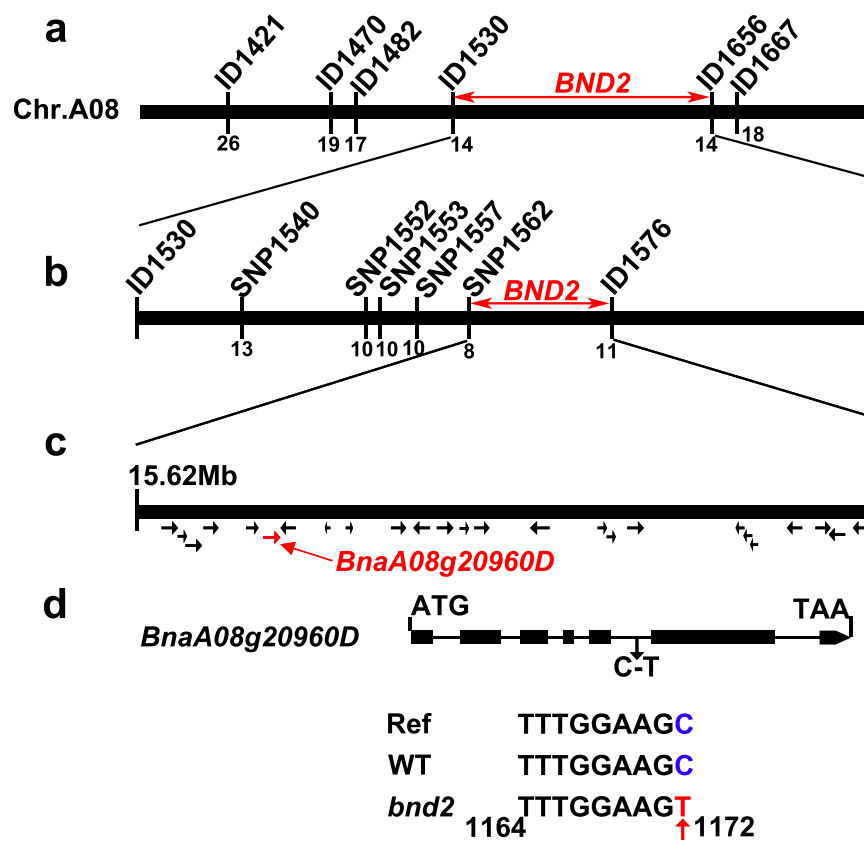

Fig. 6 Fine mapping of BND2. a The BND2 locus was primary mapped into the interval flanked by the markers ID1530 and ID1656. Numbers below each marker is the number of recombinants. b The BND2 locus was finally mapped to a 140-Kb flanked by SNP1562 and ID1576. c Relative physical position of the BND2 locus. Numbers above chromosome A08 indicate physical distance (unit: Mb). The region contains 27 annotated genes according to the 'Darmor-bzh' reference genome. The candidate gene, BnaA08g20960D is marked in red. d Structure of the BnaA08g20960D gene, a single nucleotide substitution (C-T) between bnd 2 and its wild type parent $2 \mathrm{~B}$ was identified in the fifth intron. Exons and introns were represented as black boxes or black lines, respectively

pathway of phytic acid (myo-inositol-1,2,3,4,5,6-hexakisphosphate [ $\left.\operatorname{Ins}_{6}\right]$ ]) [30]. Over the last two decades, with the discovery of IPK1 in budding yeast [31], IPK1 homologous genes were subsequently isolated from Schizosaccharomyces pombe [32], human [33], Drosophila [34], maize [30, 35], and Arabidopsis thaliana [36]. As a product of IPK1, phytic acid acts not only as a storage compound in seeds, but is also involved in hormones and signal transduction processes [37]. It has been reported that $\mathrm{InsP}_{6}$ is a specific functional co-factor of the auxin a

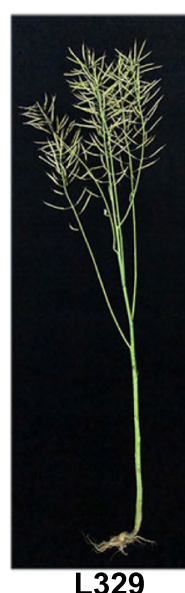

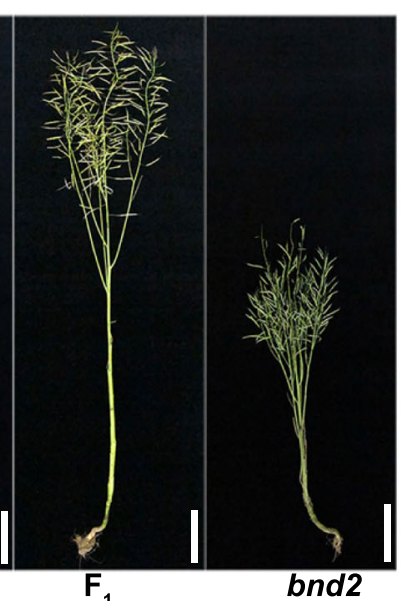

b

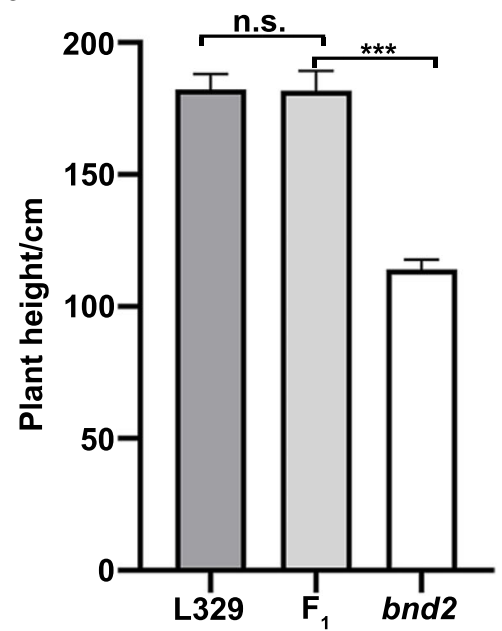

C

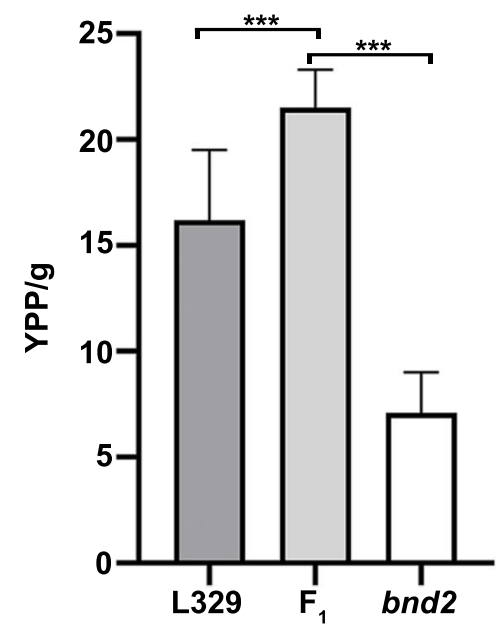

Fig. 7 Performance on plant height and grain yield of bnd2 in the hybrid line. a Phenotypes of L329 (left), bnd2 (right) and their hybrid ( $F_{1}$, middle) at the maturation stage. b-c Plant height (b) and yield per plant (c) of $L 329$, bnd2 and their $F_{1}$ hybrid at maturity. Values are shown as mean \pm SD $(n=10)$. Bars $=20 \mathrm{~cm}$. The significance of difference was determined by Student's t-test (n.s. not significant; $\left.{ }^{* * *}, P<0.001\right)$ 
receptor TIR1 [38] and acts as a 'conformational stabilizer' for TIR1 protein [39]. InsP $\mathrm{P}_{6}$ binds TIR1 and stabilizes the active amino acid residues around auxin, thus ensuring effective signal transduction between TIR1 and auxin [40]. In addition, AtIPK1 has been reported to be essential for sustaining plant growth. In Arabidopsis, the mutant atipk1-1 displayed reduced size and leaf epinasty [35], while the two mutants atipk1-2 and atipk1-3 showed more serious growth retardation [41]. Similarly, Lee et al. found that the atipk1-1 mutant was significantly smaller than the wild type (Columbia-0) [37]. The seed yield of the atipk1 mutant was only $52 \%$ of that of WT because many pods of the mutant contained abortive seeds [37]. In the present study, bnd2 showed shorter petiole leaves, decreased and shorter internodes, and decreased plant height and seed yield compared to WT (Fig. 1c-e, h-n, Additional files 2, 3, 5, and 7: Figs. S2, 3, 5, and 6, Additional file 6: Table S1), suggesting that its dwarfism may be related to auxin signal transduction, which needs to be further explored.

It has been reported that introns of many genes have a positive regulatory effect on gene expression [42]. The promoter of the rice gene $O s B P-73$ requires the participation of the gene intron sequence to drive the expression of the GUS gene in transgenic rice, but the complete OsBP-73 intron itself has no promoter activity [42]. Liu et al. [43] found that the intron of BnFAD2-C5 can enhance the transcription level of the promoter. The 5' UTR of Arabidopsis AtVTC1 contains an intron sequence, which can promote the expression of $A t V T C 1$ [44]. In addition, the existence of introns provides a variety of splicing methods for genes. The same DNA sequence can produce different protein products after mRNA transcription by alternative splicing [45]. Sun et al. [30] found that, among 18 maize full-length ZmIPK1A cDNA clones in leaves and seeds, $50 \%$ of the transcripts had interrupted reading frames due to alternative splicing of introns 6 and 7. However, intron mutations can lead to abnormal splicing, which in turn could result in exon skipping, new exon generation or intron retention [46]. Yuan et al. [47] found that a single G-A point mutation of the soybean GmIPK1 in the $5^{\prime}$ terminal of intron 5 resulted in the exclusion of the fifth exon, disrupting GmIPK1 functionality. In this study, we identified a single C-T mutation in the fifth intron of BnaA08g20960D in the dwarf mutant bnd2 (Fig. 6d), and found that the expression of BnaA08g20960D was attenuated in bnd2 (Additional file 14: Fig. S12), although no splicing change was observed between bnd2 and WT (Additional file 15: Fig. S13). We therefore considered it as the candidate gene for the dwarf phenotype of bnd2; however, the molecular basis needs to be further examined.

\section{Conclusion}

In this study, we described a new dwarf mutant bnd2 isolated using EMS mutagenesis. The mutation of BND2 decreased plant height and grain yield in the background of the inbred line, but maintained the plant height and increased grain yield in the background of the hybrid line. Through BSA-seq and fine mapping, bnd 2 was mapped to a $140.0-\mathrm{Kb}$ region on chromosome A08 in $B$. napus. In summary, we identified a dwarf mutant bnd 2 which may be useful for hybrid breeding with lodging resistance and high yield, and the fine mapping results will benefit functional analyses of genetic mechanisms of plant architecture and grain yield in rapeseed.

\section{Methods}

\section{Plant materials and growth}

$B$. napus $2 \mathrm{~B}$ was used as a wild type in this study. $2 \mathrm{~B}$ is a maintainer line of bolima cytoplasmic male sterile line. The $B$. napus dwarf mutant bnd2 was isolated and screened from $2 \mathrm{~B}$ seeds induced by $0.8 \%$ EMS solution in our previous study [19]. Another commercial cultivar L329 (Xiangyou 15) described previously [48] was used to construct the $\mathrm{F}_{2: 3}$ population and the $\mathrm{F}_{1}$ hybrid line for BND2's genetic analysis and evaluation of its potential value in hybrid breeding. Plants of all generations including their parents were grown in the filed in Ningxiang, Hunan province.

\section{Agronomic traits analysis}

Plants of all generations including their parents were grown in an irrigated field. Each plot in the field is about $2 \mathrm{~m}$ wide, $2 \mathrm{~m}$ long, with a row spacing of $33 \mathrm{~cm}$. Ten plants were planted in each row. The agronomic traits were measured and counted at maturity stage. Ten plants from plot were randomly selected for agronomic traits analysis. The plant height $(\mathrm{PH})$, internode length (IL), internode number (IN), first branch height (FBH), main inflorescence length (MIL), number of effective primary branches (NPB), number of siliques on raceme (NSR), siliques per plant (SPP), length of siliques (LS), seeds per silique (SPS), thousand-seed weight (TSW) and yield per plant (YPP), were measured and counted as previously described [49, 50]. Significant differences were determined by Student's $t$-test using SPSS version 25 (SPSS Inc., Chicago). The segregation ratio was calculated by Chi square test.

\section{Microscopy analysis}

The second internode stem segment from the top to the bottom of bnd2 and WT plants at the early stage of bolting were fixed in FAA (formalin-acetic acid-alcohol) solution for $16-20 \mathrm{~h}$, and then subjected to dehydration and transparency. The tissues were then immersed and embedded in paraffin wax (Sigma, USA), and sectioned to $6-10 \mu \mathrm{m}$ (Leica $\mathrm{rm} 2265$ ). After staining with $0.05 \%$ toluidine blue, the samples were examined and photographed by a reverse fluorescence phase contrast 
microscope (Nikon). The stem cell size and number were calculated by the Image J software (http://rsb.info. nih.gov/ij/).

\section{Genetic mapping and BSA-seq}

To map the BND2 locus, a $\mathrm{F}_{2: 3}$ mapping population containing 157 lines was obtained from the self-pollinated $\mathrm{F}_{2}$ lines, which was derived by the cross between bnd2 and L329. Young leaves were collected from each 157 $\mathrm{F}_{2: 3}$ line for genomic DNA extraction using the method of SDS extraction as described by Dellaporta et al [51]. The DNA concentration and purity were detected by Nanodrop one (Thermo Fisher, USA). The DNA of 25 extremely dwarf and 23 extremely tall individuals were mixed to make a short bulk and high bulk, separately.

Both two parents, together with above two bulks, were sequenced by next generation sequencing strategy. The paired end (PE) library was constructed according to the manufacture's instructions (NEBNext ${ }^{\circ}$ Ultra $^{\mathrm{T}}$ IIFS DNA Library Prep Kit for Illumina ${ }^{\circ}$ ), in which the genomic DNA was randomly broken into 300-500 bp fragments. High-throughput sequencing was performed on Illumina NovaSeq platform to generate average $30 \mathrm{~Gb}$ sequence data per sample. Burrows-Wheeler Alignment tool (BWA, version 0.7.15) was used to align the PE reads to the reference genome of 'Darmor-bzh' v4.1 [26]. And, SAM format was then converted to the BAM format using SAMtools (version 1.3.1). Picard tool (version 1.91) was used to sort the reads in the BAM file and remove polymerase chain reaction (PCR) duplication. Variants including single nucleotide polymorphism (SNP) and insertion/deletion (InDel) were detected by the HaplotypeCaller of Genome analysis toolkit (GATK, version 3.7). The candidate region was determined based on $\Delta$ (SNP-index) and G' value [52] calculated by QTLseqr (version 0.7.5.2) [53], and ANNOVAR (version 2016FeB1) was used to predict the effect of variants on gene function (Wuhan Genoseq Technology Co. Ltd., Hubei, China).

\section{Development of molecular markers and their genotyping}

According to the BSA-seq results and the positions of SNP and InDel on chromosomes contained in the target gene candidate region, and based on the 'Darmor- $b z h$ ' sequence of the $B$. napus reference genome, DNA sequences of SNP/InDel were extracted by extending 250 bp forward (5' end) and back ( 3 ' end) respectively, and Primer Premier (version 5.0) was used to design SNP/ InDel markers. For all markers, two parents L329 and bnd2 were used for polymorphism screening, and markers with polymorphism were used for PCR amplification and genotype identification of $\mathrm{F}_{2: 3}$ population. For InDel markers, $3 \%$ agarose gel electrophoresis was used to separate PCR products. While for SNP markers, PCR products were first identified by $1 \%$ agarose gel electrophoresis and if bands between the bnd2 and L329 were clear, then sent the PCR products to sequencing (TsingKe Biological Technology Co. Ltd., Changsha, Hunan, China). PCR sequencing results were analyzed with Sequencher (version 5.0). The band type consistent with bnd $2\left(\mathrm{P}_{1}\right)$ was recorded as $\mathrm{A}$, the band type consistent with $\mathrm{L} 329\left(\mathrm{P}_{2}\right)$ was recorded as $\mathrm{B}$, and both band types were recorded as $\mathrm{H}$, and the deletion was not recorded. The corresponding mapping markers sequences are listed in Additional file 18: Table S4.

RNA extraction and quantitative real-time PCR (qRT-PCR)

To detect mRNA expression of the bnd 2 candidate gene, BnaA08g20960D, seven-day-old seedlings of bnd2 and wild type $2 \mathrm{~B}$ grown in soil were sampled. Total RNA was extracted by AG RNAex Pro Reagent (Accurate Biology, China). The cDNA was then synthesized using the HiScript II 1st Strand cDNA Synthesis Kit (+gDNA wiper) (Vazyme, China). qRT-PCR was performed in an ABI StepOne Plus system (Thermo Fisher, USA) using ChamQ universal SYBR qPCR Master Mix (Vazyme, China) according to the manufacture's instructions. All primers are listed in (Additional file 19: Table S5) and BnActin 7 was used as an internal reference. Each experiment was biologically repeated three times.

\section{Semi-quantitative PCR (semi-qPCR) analysis}

To detect the splicing of the $b n d 2$ candidate gene, BnaA08g20960D, seven-day-old seedlings of bnd2 and wild type $2 \mathrm{~B}$ grown in soil were sampled for RNA extraction. 2xTaq Master Mix (Novoprotein, China) was used in PCR reaction according to the manufacture's instructions. PCR was performed with a 5 min denaturation at $94{ }^{\circ} \mathrm{C}$ followed by 28 (for BnActin7) or 36 (for BnaA08g20960D) cycles with each cycle composed of $94{ }^{\circ} \mathrm{C}$ for 30 s, $58^{\circ} \mathrm{C}$ for 30 s and $72^{\circ} \mathrm{C}$ for 30 s. PCR products were then analyzed by $2.0 \%$ agarose gel electrophoresis. All primers are listed in (Additional file 20: Table S6). Each experiment was biologically repeated three times.

\section{Supplementary Information}

The online version contains supplementary material available at https://doi. org/10.1186/s12870-021-02885-y.

Additional file 1: Figure S1. Phenotype of one-week-old wild type (WT) and bnd2 seedlings.

Additional file 2: Figure S2. Plants of WT and bnd2 at 5-week-old seedling stage.

Additional file 3: Figure S3. Leaves of WT and bnd2 at 5-week-old seedling stage.

Additional file 4: Figure S4. Plants of $\mathrm{WT}$ and bnd2 at peak flowering stage.

Additional file 5: Figure S5. Whole plant phenotype of WT and bnd2. 
Additional file 6: Table S1. Agronomic characters of WT and bnd2.

Additional file 7: Figure S6. Plant internodes of WT and bnd2.

Additional file 8: Figure S7. Phenotype of siliques of WT and bnd2. (DOCX $94 \mathrm{~kb}$ )

Additional file 9: Figure 58. Phenotypes of WT (left), bnd2 (right) and their $F_{1}$ hybrid (middle) at maturity.

Additional file 10: Figure S9. Plant internodes of WT (left), bnd2 (right) and their $\mathrm{F}_{1}$ hybrid (middle) at maturity.

Additional file 11: Figure S10. Phenotype and trait inheritance of bnd2 in the cross population.

Additional file 12: Table S2. Statistics of the sequencing datasets. Additional file 13: Figure S11. The genotypes of some $F_{2: 3}$ lines derived from cross between bnd2 and L329 at the marker ID1656.

Additional file 14: Figure S12. qRT-PCR analysis showing the mRNA expression levels of BnaA08g20960D in WT and bnd2.

Additional file 15: Figure S13. semi-qPCR analysis showing the mRNA expression pattern of BnaA08g20960D in WT and bnd2.

Additional file 16: Figure S14. Phenotypes of $L 329$ (left), bnd2 (right) and their hybrid ( $F_{1}$, middle) at the maturation stage.

Additional file 17: Table S3. Agronomic characters of heterozygous $F_{1}$ between $L 329$ and bnd2.

Additional file 18: Table S4. Primer sequences for designed markers.

Additional file 19: Table S5. Primer sequences for qRT-PCR.

Additional file 20: Table S6. Primer sequences for semi-qPCR.

\section{Abbreviations}

BND2: Brassica napus dwarf 2; EMS: ethyl methanesulfonate; BSA: bulked segregant analysis; BSA-seq: BSA and Next generation sequencing; SNP(s): single nucleotide polymorphism(s); InDel(s): insertion(s) and deletion(s); YPP: yield per plant; SPS: seeds per silique; SPP: siliques per plant; PH: plant height; IL: internode length; FBH: first branch height; MIL: main inflorescence length; NPB: number of effective primary branches; NSR: number of siliques on raceme; LS: length of siliques; TSW: thousandseed weight; PCR: polymerase chain reaction; IPK1: Inositol 1,3,4,5,6Pentapentaphosphate 2 kinase 1

\section{Acknowledgements}

We thank Dr. Zhongsong Liu for providing L329 (xiangyou 15) seeds, Prof. Wusheng Peng for $2 \mathrm{~B}$ seeds.

\section{Authors' contributions}

$X L, F X, D M$ and $X Z$ designed and carried out the research. $X L$ and FX performed the experiments. $W Z, J Y, X L, M Z, P Y$ provided technical assistance to $X L$ and $F X$. XL, CC, $X L, D M$ and $X Z$ analyzed the data. $X L$ wrote the manuscript. $C C, X L, D M$ and $X Z$ revised the manuscript. All authors read and approved.

\section{Funding}

This work was supported by the Basic Research Program of Shenzhen Municipal Science and Technology Innovation Committee (No. JCYJ20170818112212721), the Basic Research Program of Changsha Municipal Science and Technology (No. kq1901028), and the Natural Science Foundation of Hunan province (No. 2018JJ3036). All the funders only provided funds but did not take participation in the design of the study and collection, analysis, and interpretation of data and in writing the manuscript.

\section{Availability of data and materials}

All data generated or analyzed during this study are included in this published article (and its additional files). The original sequencing datasets of BSA-seq for bnd2 have been deposited in the NCBI Sequence Read Archive (SRA, https://www.ncbi.nlm.nih.gov/sra) under accession numbers: SRR12968239, SRR12968240, SRR12968241, and SRR12968242. Any material generated during the current study is available from the corresponding author on reasonable request.
Ethics approval and consent to participate

Not applicable.

\section{Consent for publication}

Not applicable.

\section{Competing interests}

The authors declare that they have no competing interests.

\section{Author details}

${ }^{1}$ College of Biology, Hunan Hybrid Rape Engineering and Technology Research Center, Hunan University, Changsha 410082, China. ${ }^{2}$ Shenzhen Institute, Hunan University, Shenzhen 518057, China. ${ }^{3}$ Key Laboratory of Agro-Ecological Processes in Subtropical Region, Institute of Subtropical Agriculture, Chinese Academy of Sciences, Changsha 410125, China. ${ }^{4}$ College of Agronnomy, Hunan Agricultural University, Changsha 410128, China.

Received: 6 August 2020 Accepted: 10 February 2021

Published online: 26 February 2021

\section{References}

1. Zhu DJ, Zhang H, Huang $H$, Ning WY, Zhang YC. Effects of different fertilization treatments on yield and economic benefits of rape at different soil fertility levels. Jiangsu Agricultural Science. 2013;41(10):73-6.

2. Wang X, Wang H, Wang J, Sun R, Wu J, Liu S, Bai Y, Mun JH, Bancroft I, Cheng F, et al. The genome of the mesopolyploid crop species Brassica rapa. Nat Genet. 2011;43(10):1035-40.

3. Bayer PE, Hurgobin B, Golicz AA, Chan CK, Yuan Y, Lee HT, Renton M, Meng J, Li R, Long Y, Zou J, Bancroft I, Chalhoub B, King GJ, Batley J, Edwards D. Assembly and comparison of two closely related Brassica napus genomes. Plant Biotechnol J. 2017;15(12):1602-10.

4. Foisset $N$, Delourme R, Barret $P$, Renard M. Molecular tagging of the dwarf BREIZH (Bzh) gene in Brassica napus. Theor Appl Genet. 1995;91(5):756-61.

5. Zeng, XH: Comparing effrctiveness of different mutagens for seed quality and analysis of mutants in Brassica napus. http://www.hzau.edu.cn/ (2010). Accessed 1 Dec 2010.

6. Liu HL. Cultivation of edible rape. Shanghai: Shanghai Scientific \& Technical Publishers; 1987.

7. Wang Y, Chen W, Chu P, Wan S, Yang M, Wang M. Mapping a major QTL responsible for dwarf architecture in Brassica napus using a singlenucleotide polymorphism marker approach. BMC Plant Biol. 2016;16(1):178.

8. Wang M, Zhao Y, Chen F, Yin XC. Inheritance and potentials of a mutated dwarfing gene ndf1 in Brassica napus. Plant Breed. 2004;123(5):449-53.

9. Li Y, Fu SH, Yang J, Wang JS, Zhou Q, Chen XH, Tao LR, Kang ZM, Tang R, Zhang RQ. The identification and application of dwarf mutation bndf-1 in Brassica napus. Chinese Agricultural Science Bulletin. 2013;29(13):173-7.

10. Liu C, Wu JS. Subcellular localization of semi-dwarf gene BnaA6. rga-ds and identification of its transgenic Brassica napus L. Chinese J Oil Crop Sciences. 2015;37(4):427-32.

11. Song X, Pu DF, Tian $L S$, Yu QQ, Yang YH, Dai BB, Zhao CB, Huang CY, Deng WM. Genetic analysis and characterization of hormone response of semidwarf mutant $d w-1$ in Brasscia napus L. Sci Agric Sin. 2019;52(10):1667-77.

12. Xiang Y, Tong C, Yu S, Zhang T, Zhao J, Lei S, Du C, Liu S. Genetic segregation analysis of a rapeseed dwarf mutant. Pakistan J Bot. 2016;48(4): 1629-35.

13. Zhao B, Li H, Li J, Wang B, Dai C, Wang J, Liu K. Brassica napus DS-3, encoding a DELLA protein, negatively regulates stem elongation through gibberellin signaling pathway. Theor Appl Genet. 2017;130(4):727-41.

14. Zhang RM, Li C, Chen DL, Xiang Y. Breeding of short stem, erect plant type DW 871 in Brassica napus L. Seed. 2019;38(2):116-23.

15. Li H, Li J, Song J, Zhao B, Guo C, Wang B, Zhang Q, Wang J, King GJ, Liu K. An auxin signaling gene BnaA3.IAA7 contributes to improved plant architecture and yield heterosis in rapeseed. New Phytol 2019; 222(2):837-851.

16. Wang $Y$, He J, Yang L, Wang Y, Chen W, Wan S, Chu P, Guan R. Fine mapping of a major locus controlling plant height using a high-density single-nucleotide polymorphism map in Brassica napus. Theor Appl Genet. 2016;129(8):1479-91.

17. Zhao B, Wang B, Li Z, Guo T, Zhao J, Guan Z, Liu K. Identification and characterization of a new dwarf locus DS-4 encoding an aux/IAA7 protein in Brassica napus. Theor Appl Genet. 2019;132(5):1435-49. 
18. Cheng H, Jin F, Zaman QU, Ding B, Hao M, Wang Y, Huang Y, Wells R, Dong Y, Hu Q. Identification of Bna.IAA7.C05 as allelic gene for dwarf mutant generated from tissue culture in oilseed rape. BMC Plant Biol. 2019;19:500

19. Liao XY, Yan JD, Zhong M, Zhuo YH, Wu D, He RQ, Zhao XY, Liu XM. EMS mutagenesis and analysis of multi-branched and long-silique mutants in Brassica napus L. Life Science Research. 2016;20(5):435-41.

20. Michelmore RW, Paran I, Kesseli RV. Identification of markers linked to disease-resistance genes by bulked segregant analysis: a rapid method to detect markers in specific genomic regions by using segregating populations. Proc Natl Acad Sci U S A. 1991;88(21):9828-32.

21. Zou C, Wang P, Xu Y. Bulked sample analysis in genetics, genomics and crop improvement. Plant Biotechnol J. 2016;14(10):1941-55.

22. Yang Z, Huang D, Tang W, Zheng Y, Liang K, Cutler AJ, Wu W. Mapping of quantitative trait loci underlying cold tolerance in rice seedlings via highthroughput sequencing of pooled extremes. PLoS One. 2013;8(7):e68433.

23. Sun J, Yang L, Wang J, Liu $H$, Zheng $H$, Xie D, Zhang $M$, Feng $M$, Jia $Y$, Zhao $\mathrm{H}$, Zou D. Identification of a cold-tolerant locus in rice (Oryza sativa L.) using bulked segregant analysis with a next-generation sequencing strategy. Rice. 2018;11(1):24.

24. Kaminski KP, Kørup K, Andersen MN, Sønderkær M, Andersen MS, Kirk HG, Nielsen KL. Next generation sequencing bulk segregant analysis of potato support that differential flux into the cholesterol and stigmasterol metabolite pools is important for steroidal glycoalkaloid content. Potato Res. 2016;59(1):81-97.

25. Song J, Li Z, Liu Z, Guo Y, Qiu LJ. Next-generation sequencing from bulkedsegregant analysis accelerates the simultaneous identification of two qualitative genes in soybean. Front Plant Sci. 2017;8:919.

26. Chalhoub B, Denoeud F, Liu S, Parkin IAP, Tang H, Wang X, Chiquet J, Belcram $H$, Tong C, Samans B, et al. Early allopolyploid evolution in the post-neolithic Brassica napus oilseed genome. Science. 2014;345(6199):950-3.

27. Hedden P. The genes of the green revolution. Trends Genet. 2003;19(1):5-9.

28. Ma N, Li L, Xu J, Li J, Yu LP, Li GM, Zhang CL. Researches on the lodging resistance and agronomic traits of winter rape (Brassica napus L.). Crops. 2010;6(6):36-41.

29. Muangprom A, Mauriera I, Osborn TC. Transfer of a dwarf gene from Brassica rapa to oilseed $B$. napus, effects on agronomic traits, and development of a 'perfect' marker for selection. Mol Breeding. 2006;17(2): 101-10.

30. Sun Y, Thompson M, Lin G, Butler H, Gao Z, Thornburgh S, Yau K, Smith DA, Shukla VK. Inositol 1,3,4,5,6-pentakisphosphate 2-kinase from maize: molecular and biochemical characterization. Plant Physiol. 2007;144(3):1278-91.

31. York JD, Odom AR, Murphy R, Ives EB, Wente SR. A phospholipase Cdependent inositol polyphosphate kinase pathway required for efficient messenger RNA export. Science. 1999;285:96-100.

32. Ives EB, Nichols J, Wente SR, York JD. Biochemical and functional characterization of inositol 1,3,4,5,6-pentakisphosphate 2-kinases. J Biol Chem. 2000;275(47):36575-83.

33. Verbsky JW, Wilson MP, Kisseleva MV, Majerus PW, Wente SR. The synthesis of inositol hexakisphosphate: characterization of human inositol 1,3,4,5,6pentakisphosphate 2-kinase. J Biol Chem. 2002;277(35):31857-62.

34. Seeds AM, Sandquist JC, Spana EP, York JD. A molecular basis for inositol polyphosphate synthesis in Drosophila melanogaster. J Biol Chem. 2004; 279(45):47222-32

35. Stevenson-Paulik J, Bastidas RJ, Chiou ST, Frye RA, York JD. Generation of phytate-free seeds in Arabidopsis through disruption of inositol polyphosphate kinases. Proc Natl Acad Sci U S A. 2005;102(35):12612-7.

36. Sweetman D, Johnson S, Caddick SEK, Hanke DE, Brearley CA. Characterization of an Arabidopsis inositol 1,3,4,5,6-pentakisphosphate 2kinase (AtIPK1). Biochem J. 2006;394(1):95-103.

37. Lee HS, Lee DH, Cho HK, Kim SH, Auh JH, Pai HS. InsP $\mathrm{P}_{6}$-sensitive variants of the Gle1 mRNA export factor rescue growth and fertility defects of the ipk1 low-phytic-acid mutation in Arabidopsis. Plant Cell. 2015;27(2):417-31.

38. Tan X, Calderon-villalobos LIA, Sharon M, Zheng C, Robinson CV, Estelle M, Zheng N. Mechanism of auxin perception by the TIR1 ubiquitin ligase. Nature. 2007;446(7136):640-5

39. Hao GF, Yang GF. The role of Phe 82 and Phe351 in auxin-induced substrate perception by TIR1 ubiquitin ligase: a novel insight from molecular dynamics simulations. PLoS One. 2010;5(5):e10742.

40. Jia XB, Zhang Y, Sun HW, Chen L, Shen RX, Lai CM. Molecular dynamics simulation study on the interaction mode of auxin perception. Acta Chimica Sin. 2010;68(24):2500-8.
41. Kuo HF, Chang TY, Chiang SF, Di Wang W, Charng YY, Chiou TJ. Arabidopsis inositol pentakisphosphate 2-kinase, AtIPK1, is required for growth and modulates phosphate homeostasis at the transcriptional level. Plant J. 2014; 80(3):503-15.

42. Chen J, Wang ZY. Expression of OsBP-73 gene requires involvement of its intron in rice. J Plant Physiol Mol Biol. 2004;30(1):81-6.

43. Liu RY, Liu F, Zhang ZQ, Guan CY. Functional analysis of BnFAD2-C5 promoter and intron at expression level in Brassica napus. Acta Agron Sin. 2016;42(10):1471-8.

44. Li SH, Wang FR, Huang RF, Dong JG, Wang J. Function analysis of VTC1 5' UTR intron in Arabidopsis. J Agric Sci and Tech. 2016;18(6):52-7.

45. Ner-gaon H, Halachmi R, Savaldi-goldstein S, Rubin E, Ophir R, Fluhr R Intron retention is a major phenomenon in alternative splicing in Arabidopsis. Plant J. 2004;39(6):877-85.

46. Nakai $\mathrm{K}$, Sakamoto $\mathrm{H}$. Construction of a novel database containing aberrant splicing mutations of mammalian genes. Gene. 1994;141(2):171-7.

47. Yuan FJ, Zhu DH, Tan YY, Dong DK, Fu XJ, Zhu SL, Li BQ, Shu QY. Identification and characterization of the soybean IPK1 ortholog of a low phytic acid mutant reveals an exon-excluding splice-site mutation. Theor Appl Genet. 2012;125(7):1413-23.

48. Zhang ZQ, Xiao G, Liu RY, Tan TL, Guan CY, Wang GH, Chen SY, Wu XM, Guan M, Li Q. Proteomic analysis of differentially expressed proteins between Xiangyou 15 variety and the mutant M15. Front Biol. 2014;9(3): 234-43.

49. Zhou B, Lin J, Peng W, Peng D, Zhuo Y, Zhu D, Huang X, Tang D, Guo M, He R, Zhang J, Li X, Zhao X, Liu X. Dwarfism in Brassica napus L. induced by the over-expression of a gibberellin 2-oxidase gene from Arabidopsis thaliana. Mol Breeding. 2012;29(1):115-27.

50. Yang P, Li Y, He C, Yan J, Zhang W, Li X, Xiang F, Zuo Z, Li X, Zhu Y, Liu X. Phenotype and TMT-based quantitative proteomics analysis of Brassica napus reveals new insight into chlorophyll synthesis and chloroplast structure. J Proteome. 2020;214:103621.

51. Dellaporta SL, Wood J, Hicks JB. A plant DNA minipreparation: version II. Plant Mol Biol Report. 1983;1(4):19-21.

52. Magwene PM, Willis JH, Kelly JK. The statistics of bulk segregant analysis using next generation sequencing. PLoS Comput Biol. 2011;7(11):1-9.

53. Mansfeld BN, Grumet R. QTLsegr: an R package for bulk segregant analysis with next-generation sequencing. Plant Genome. 2018;11(2):1-5.

\section{Publisher's Note}

Springer Nature remains neutral with regard to jurisdictional claims in published maps and institutional affiliations.

Ready to submit your research? Choose BMC and benefit from:

- fast, convenient online submission

- thorough peer review by experienced researchers in your field

- rapid publication on acceptance

- support for research data, including large and complex data types

- gold Open Access which fosters wider collaboration and increased citations

- maximum visibility for your research: over $100 \mathrm{M}$ website views per year

At $\mathrm{BMC}$, research is always in progress.

Learn more biomedcentral.com/submissions 